the couch ready for the exposure to be made. Further films are made at thirty and fifty minutes after the injection. The chief difficulty with which the radiologist has to contend is the presence of gas collection in the alimentary tract. This gives rise to more trouble in children than it does in adults, especially when they are confined to bed. No method of special preparation has been of much use in my experienceaperients and enemata seem often to make it worse. Nothing does so much good as allowing the child to be up and about when this is pessible. The method I find most effective is to give a dose of pulv. glyc. co. forty-eight hours before the injection, and if possible to have the child up and about. When the preliminary film shows large gas collections in spite of this preparation I wait, if possible, a few days and try again, but if the gas collection persists I do not now regard it as a contraindication to proceeding with the injection, as one can often arrive at a diagnosis in spite of the gas collection, and as the injection causes so little inconvenience to the patient, it is always worth while making the attempt. A recent innovation in the examination of young children which I have found to be of considerable value is the use of the Lysholm fixed Bucky grid. The faint lines of this grid do not detract from the diagnostic value of the films, and it enables one to obtain the advantages of a film made with a Potter-Bucky diaphragm without entailing the disadvantages which are associated with the longer exposure required to allow time for the grid to move. The exposures can be made in a fraction of a second, an enormous advantage when one is dealing with infants and small children.

\section{USES AND INDICATIONS}

The indications for intravenous urography in children are much the same as those for which it is used in adults. It can be employed whenever we wish to visualize the urinary tract, while it will also give certain information with regard to kidney function and the dynamics of the urinary tract. I have found it extremely useful in chilc ren for the purpose of localizing doubtful shadows in the region of the kidneys and ureters. Owing to the fact that the pelvic shadow is not so dense as that obtained with retrograde pyelography the outline of a stone may be seen through the shadow of the uroselectan, thus rendering its localization more easy. In the case of doubtful shadows which are approximately in the line of the ureter, while it is true that one seldom sees the ureter filled throughout the whole of its length in any one film, there is almost always one film in the series which shows that portion of the ureter filled which is in the region of the doubtful shadow.

It is, however, in the investigation of cases of chronic pyuria that intravenous urography has been found if particular value in children. Ordinary medical treatment cannot hope to bring about a cure in these cases when there is an associated congenital deformity in the urinary tract. Such deformities would appear to be much more common than was at one time supposed. My colleague Dr. Neale found congenital abnormalities in five out of a hundred consecutive necropsies in children. He takes the view that while there is no useful purpose to be served by making an intravenous urogram in cases of pyuria which clear up quickly, this method of investigation should be used in all cases which do not respond to medical treatment in a reasonable time.

Previously the ordinary radiographic examination of the urinary tract could be supplemented by cystography and retrograde pyelography. Cystography is a simple procedure and presents no difficulty, but unfortunately the abnormalities in these cases of chronic pyuria are almost always in the upper portion of the urinary tract, and while occasionally one can demonstrate hydroureter and hydronephrosis by the cystographic method, it is not possible as a general. rule. For the reasons stated retrograde pyelography is not a practical routine method of investigation. The introduction of intravenous urography has, however, revolutionized the investigation of these cases. Its simplicity and safety allow of its being used in all cases. By this method one can demonstrate congenital abnormalities when they exist, and can also show whether the trouble is unilateral or bilateral-a most important matter to decide from the point of view of treatment, since in unilateral cases surgical treatment is indicated. The most common abnormality seen is hydronephrosis, which may or may not be associated with a condition of hydroureter. In no case of chronic pyuria sent for examination has the intravenous method failed to demonstrate an abnormality in the urinary tract, and an analysis of a series of cases which were sent for investigation, although they had responded to treatment and been cured, showed that in every one the report on the urograms was that they were normal. Intravenous urography, therefore, is of the greatest possible value in infants and children for the reason that in them, for the first time, it has provided a practical routine method of visualizing the upper urinary tract.

I should like to express my thanks to my colleague, Dr. Thorpe, and to the sister in charge of the $x$-ray department at the Children's Hospital, Birmingham, for their help in connexion with this investigation.

Acta Rad., 1931, xii, 65

BiBLIOGRAPHY

Brasch, W. F.: Journ. Urol., 1931, xxv.

Bugbee, H. (i., and Murphy, B. J.: Ibid., 1931, xxv.

Herbst, R. H. Ibid, 1931, xxv.

Neale, A. V.: Arch. Dis. in Child., 1931, vi.

Sirre, Gynec and Obstet. September, 1930.

von Lichtenberg, A.: Journ. Urol., 1931, xxv.

\section{A CASE OF SYPHILIS (GUMMATA) OF THE HEART}

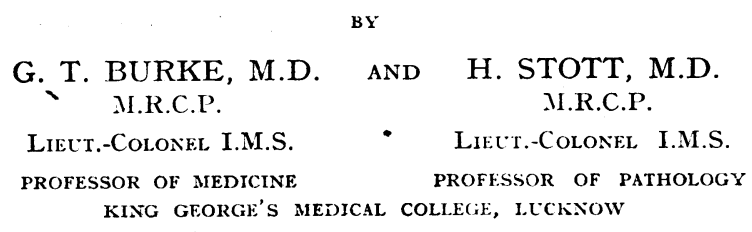

Myocardial syphilis is generally recognized as occurring either as a few large scattered gummata or as small diffuse gummata scattered as an infiltration throughout the musculature, especially in the septum and in the region of the bundle of $\mathrm{His}$. The large gummata constitute, as in other organs, the real specific naked-eye lesion, of which Dieulafoy's description can hardly be improved:

“Their yellowish appearance, their elastic consistency, their well-defined limits, their multiplicity, or the coexistence of miliary gummata in the neighbourhood can scarcely allow any mistake. In some cases they are surrounded by healthy muscle tissue, and project towards the endocardium or towards the pericardium. The serous membranes covering them are thickened, and the layers of pericardium may be adherent. In other cases the endocardium is not thickened but ulcerated, the gumma having emptied itself into the ventricular cavity, which has become hollowed out at the expense of the myocardium, with the formation of partial aneurysm."

Projecting cardiac gummata are distinctly rare: when recorded they have been into the ventricular cavity. No case of auricular gummata has been noted in a survey of the literature available in Lucknow. In the case reported below the degree of obstruction to the blood in the superior vena cava was remarkable, producing great dilatation of that vessel, and marked head and neck oedema. The sudden death was apparently due to a thrombosis of the slowed blood flow in the large vein. No gummata were found in the neighbourhood of the bundle of His, although from the characters of the pulse it would not have been surprising to have found them. 
CAse Record

Lokhe, a Hindu male, aged 40, agricultural labourer, was admitted to hospital on December 15th, 1931, with complaints of discomfort in the abdomen and difficulty in respiration for about two and a half months, and a history of cough with expectoration for one and a half years.

He was suffering from dyspnoea-not usually amounting to orthopnoea-pulmonary congestion, enlarged liver, and ocdema. Although the heart was not enlarged there was myocardial weakness, the cardiac impulse being scarcely palpable and the sounds very poor. There was no murmur audible, and the pulse rate was 70 and below, with a regular rhythm. The arterial walls were soft and the pulse tension low. Beyond the congestion there were no pulmonary signs. The liver was moderately enlarged, firm, and tender. Pyorrhoea, lack of appetite, dyspepsia, and constipation were present. There was no ascites, and the spleen was not palpable. Oedema was to some extent general, but there was a much greater degree over the chest, arms, head, and neck than in the lower part of the body; the veins in the neck and the upper part of the chest were distended. The Wassermann reaction of the blood was completely positive. $X$-ray examination of the chest showed no indication of mediastinal growth, though the hilum shadows were prominent, especially on the right side.

It was considered that he had some obstruction to the superior vena cava, probably syphilitic, although the $x$-ray picture did not give much support to a diagnosis of syphilitic mediastinitis. The myocardial affection was thought to be undoubtedly syphilitic. Treatment by potassium iodide was initiated at once.

The oedema, especially of the face, became increasingly more obvious, and the pulse rate gradually slower. A partial heart-block appeared, many beats being dropped. The patient died quite suddenly on December 30th.

\section{Post-MORTEM EXAMINATION}

Oedema occurred in the lower limbs and round the ankles, but was present to a far greater degree over the head and neck, chest, and arms. No ascites. There were several ounces of blood-stained serum in both pleural cavities.

Pericardium.-The outer layer was thickened and adherent to the surrounding structures, especially round the termination of the superior vena cava and commencement of the aorta, which were intimately matted together. The visceral pericardium was free. The pericardial sac contained half an ounce of fluid.

Heart.-Size, small. The right auricle was normal externally, but its size was increased. On opening it from the front three globular, light grey gummata were observed, each about the size of a thumb-nail, projecting on a common base from the posterior wall of the auricle and slightly adherent to the anterior wall. They presented the appear ance of small birds' eggs in an auricular nest. The gummata much obstructed the orifice of the superior vena cava, and, to a lesser degree, that of the inferior vena cava. Microscopical section showed gummatous structure. The right ventricle showed a fourth gumma immediately below the right auricle at the upper end of the right ventricular border. The left auricle was normal. The left ventricle showed some linear fibrosis in the myocardium. Microscopically there was congestion of coronary veins and slight fibrosis.

Aorta.-There were raised patches and slight scarring of syphilitic aortitis. The coronary arteries were patent, aortic and other valves normal.

Superior Vena Cava.-The wall was markedly thickened and oedematous, and dilated for the last six inches before its opening into the auricle. Endothelium was roughened and covered with greyish ante-mortem clot, the lumen being finally obliterated with red clot, which was the immediate cause of sudden death. The opening of the vein into the right auricle, as seen from the venous side, was almost obliterated by the round gummata which nearly filled the right auricular cavity. Blood could pass to the ventricle only by coursing over and around these gummata.

Inferior Vena Cava.-Similar changes were present, but to a lesser degree.

Liver.-This weighed $35 \mathrm{oz}$. Microscopically there were small areas of lymphocytic infiltration and venous congestion, but no necrosis or fibrosis.
Left Pleural Cavity and Lung.-The upper and front part of the cavity showed a cherry-sized nodule arising from the rib periosteum and penetrating the tissue of the anterior edge of the upper lobe of the left lung. The edge of this nodule was sharply defined and on micrcscopical section showed a gummatous structure.

Right Pleural Cavity and Lung.-This showed old firm adhesions along the posterior edge and at the base. An old calcareous nodule was present at the apex and one in the middle lobe-probably tuberculous.

Kidneys.-Weight, 3 and 2 oz.-normal.

Spleen.-Weight, $2 \frac{1}{2}$ oz.-normal.

No obvious abnormality was discovered elsewhere, and the liver, kidney, and spleen showed no amyloid change.

\section{THE PRINCIPLES AND METHODS OF MALARIA CONTROL IN ASSAM* $\mathrm{BY}$}

G. C. RAMSAY, O.B.E., M.D., D.T.M. \& H.

PRINCIPAL, INDIA BRANCI,, THE ROSS INSTITUTE, LONDON AND

J. DE la M. SAVAGE, M.A., B.M., B.CH.

MALARIA RESEARCH OFFICER, ASSAM MEDICAL RESEARCH SOCIETY, PASTEUR INSTITUTE, SHILLONG, ASSAMI

The object of this paper is to state the guiding princip'es for the control of malaria in Assam, and, as far as possible, the methods of control which we advocate. It is unnecessary here to emphasize the importance of control of a disease which causes a greater amount of sickness, inefficiency, and mortality than any other disease in the world, and the ravages of which have formed one of the greatest obstacles to colonization and civilization. Malaria is undoubtedly Assam's greatest problem, and has cost the local Government and the industries many crores of rupees.

Of the measures directed against adult mosquitos and their bites, nets, mosquito-boots, and screening are common-sense precautions ; they are cheap and effective, and might be more widely used. Electric fans, punkahs, and repellents such, as kerosene, citronella, and preparations containing pyrethrum are of use, as are sprays, discriminate jungle clearing, and animal prophylaxis. Jungle and other vegetation, provided it be not densely shading a malariogenic channel or swamp, may be cleared on the assumption that it affords shelter to anophelinae during the day time. The importance of animal prophylaxis is noticeable in species such as $A$. maculatus, $A$. aconitus, and $A$. philippinensis, which are known to be transmitters of human malaria when animal blood is not to any extent available. A. minimus is a " human blood lover," and the presence of animals, such as cattle and goats, is not, therefore, of much practical value.

Hand eatching of adults is worth while in certain areas if it can be properly organized. Systematically performed, it reduces the number of infected adults, and is a valuable method for bungalows, hospitals, and jails. The establishment of adult " catching stations" is also a most useful method of testing the efficacy of anti-larval measures. "Catching stations" are selected shelters, preferably human habitations, where regular catches of anophelinae are made by the same collector for a stated time. This method frequently leads to the detection of dangerous breeding places which might otherwise be overlooked.

It will be seen that measures directed against adult mosquitos have a limited application as far as tea-garden labour forces or villagers are concerned. Measures directed against the larvae of malaria-transmitting mosquitos are, however, of more general application.

It has been proved that $A$. minimus is practically entirely responsible for the transmission of malaria in Assam. We have found that this species in nature breeds under certain defined conditions.

* An address to the Assam Branch of the British Medical Association, April, 1932 